\title{
Vitamin B12 measurements across neurodegenerative disorders
}

\author{
Nijee S. Luthra ${ }^{1 *}$, Ariane H. Marcus ${ }^{2}$, Nancy K. Hills ${ }^{3}$ and Chadwick W. Christine ${ }^{2}$
}

\begin{abstract}
Background: Vitamin B12 deficiency causes a number of neurological features including cognitive and psychiatric disturbances, gait instability, neuropathy, and autonomic dysfunction. Clinical recognition of B12 deficiency in neurodegenerative disorders is more challenging because it causes defects that overlap with expected disease progression. We sought to determine whether B12 levels at the time of diagnosis in patients with Parkinson's disease (PD) differed from those in patients with other neurodegenerative disorders.

Methods: We performed a cross-sectional analysis of B12 levels obtained around the time of diagnosis in patients with PD, Multiple System Atrophy (MSA), Dementia with Lewy Bodies (DLB), Alzheimer's disease (AD), Progressive Supranuclear Palsy (PSP), Frontotemporal Dementia (FTD), or Mild Cognitive Impairment (MCI). We also evaluated the rate of B12 decline in $\mathrm{PD}, \mathrm{AD}$, and $\mathrm{MCl}$.
\end{abstract}

Results: In multivariable analysis adjusted for age, sex, and B12 supplementation, we found that B12 levels were significantly lower at time of diagnosis in patients with PD than in patients with PSP, FTD, and DLB. In PD, AD, and MCl, the rate of B12 decline ranged from -17 to $-47 \mathrm{pg} / \mathrm{ml} / \mathrm{year}$, much greater than that reported for the elderly population.

Conclusions: Further studies are needed to determine whether comorbid B12 deficiency affects progression of these disorders.

Keywords: Vitamin B12, Cyanocobalamin, Subacute combined degeneration

\section{Background}

Vitamin B12 deficiency is common in aging and causes a number of neurological conditions including cognitive and psychiatric disturbances, gait instability, neuropathy, and autonomic dysfunction [1-5]. Because features of B12 deficiency overlap with those seen in neurodegenerative diseases, clinical recognition of this deficiency is difficult and requires blood measurement.

We recently measured B12 levels at baseline in early, untreated Parkinson's disease (PD) patients from the DATATOP study [6] and found that 5\% had deficient level $(<212$

\footnotetext{
* Correspondence: nijee.luthra@ucsf.edu

'Department of Neurology, University of California, San Francisco, 1635 Divisadero, Suite 520-530, San Francisco, CA 94115, USA

Full list of author information is available at the end of the article
}

$\mathrm{pg} / \mathrm{ml})$ and $13 \%$ had borderline low level $(<250 \mathrm{pg} / \mathrm{ml})$ [7]. We also found that those participants in the lowest B12 tertile had greater annualized worsening of gait and balance as assessed by the ambulatory capacity score compared to those in the middle or upper B12 tertiles [8]. B12 levels, but not homocysteine, methylmalonic acid, or holotranscobalamin, were associated with greater gait impairment. Because the DATATOP study was conducted in the late 1980's, it is not known if these rates are accurate in contemporary patients.

Since in our prior study hematological indices (mean corpuscular volume and hematocrit) did not help identify patients with low B12 levels, knowledge of B12 levels among neurodegenerative conditions could increase the diagnostic rate of B12 insufficiency in patients with PD and thus allow for appropriate treatment. In this exploratory study, we retrospectively analyzed B12 measurements across various neurodegenerative diseases obtained around the time of

(c) The Author(s). 2020 Open Access This article is licensed under a Creative Commons Attribution 4.0 International License, which permits use, sharing, adaptation, distribution and reproduction in any medium or format, as long as you give appropriate credit to the original author(s) and the source, provide a link to the Creative Commons licence, and indicate if changes were made. The images or other third party material in this article are included in the article's Creative Commons licence, unless indicated otherwise in a credit line to the material. If material is not included in the article's Creative Commons licence and your intended use is not permitted by statutory regulation or exceeds the permitted use, you will need to obtain permission directly from the copyright holder. To view a copy of this licence, visit http://creativecommons.org/licenses/by/4.0/ The Creative Commons Public Domain Dedication waiver (http://creativecommons.org/publicdomain/zero/1.0/) applies to the data made available in this article, unless otherwise stated in a credit line to the data. 
diagnosis and evaluated the rate of B12 decline in patients with PD compared to patients with a variety of other neurodegenerative disorders.

\section{Methods}

\section{Study design and protocol}

The study was approved by Committee on Human Research at University of California, San Francisco (UCSF). We searched electronic medical records between 2007 and 2015 to identify all patients $>40$ years old seen in the UCSF Department of Neurology with a recorded B12 measurement, obtained as part of routine clinical care, at the UCSF Clinical laboratory. During the timeframe of 2007-15, the UCSF laboratory used the same Vitamin B12 Chemiluminescent Assay (ADVIA Centaur System, Siemens). At the low end of the normal range $207 \mathrm{pg} / \mathrm{ml}$, this assay has a $4 \%$ within-run coefficient of variation $(\mathrm{CV})$ and a $4.4 \%$ run-to-run $\mathrm{CV}$. In order to obtain an estimate of endogenous B12 levels and because levels $>1999 \mathrm{pg} / \mathrm{ml}$ are not quantified and only resulted as such, we excluded patients with levels greater than the UCSF laboratory upper limit of normal (>911 $\mathrm{pg} / \mathrm{ml}$ ) because these high levels likely reflected excessive supplementation of B12. As clinical B12 deficiency may occur at or above the laboratory cutoff $(211 \mathrm{pg} / \mathrm{ml})$, we used a cut-off of $<250 \mathrm{pg} / \mathrm{ml}$ to identify patients at higher risk for developing B12 deficiency [7]. Only patients with a B12 measurement within \pm 3 years of neurodegenerative disease diagnosis were included in the analysis. Remaining records were reviewed to confirm the year of diagnosis and if and when levodopa was started. Because treatment with metformin and proton pump inhibitors are associated with reduced B12 levels while treatment with $\mathrm{B} 12$ and/or a multivitamin increases B12 levels, we reviewed the medical record to determine whether patients had these exposures documented at the time of the blood draw for B12.

We included the following diagnoses: $\mathrm{PD}, \mathrm{AD}, \mathrm{MSA}$, DLB, PSP, FTD and MCI. Patients were categorized according to the diagnosis they received at their latest neurology visit. In order to develop cohorts with as typical a clinical phenotype as possible, those with a diagnosis of mixed dementia (i.e. vascular dementia plus AD) were excluded. To evaluate the rate of B12 decline (change in B12 levels/year), we identified patients diagnosed with $\mathrm{PD}, \mathrm{AD}$, or $\mathrm{MCI}$ and who had at least two B12 measurements $>6$ months apart, with the first measurement within 3 years of diagnosis. Since B12 levels in the elderly have been shown to gradually decline over time [9] and in order to estimate the endogenous rate of decline of $\mathrm{B} 12$, we excluded patients with $>10 \%$ increase between first and second measurements since such an increase would likely reflect that the patient had begun B12 supplementation between measurements.

\section{Statistical analysis}

Statistical analyses were performed using Stata version 15.1 (StataCorp, College Station, TX). Patient characteristics across diagnosis groups were compared using Fisher's exact tests for categorical variables and analysis of variance (ANOVA) for continuous variables. We first examined covariates potentially associated with B12 levels using univariate linear regression models. Variables significant at the 0.05 level in univariate analysis were then included in a multivariable model. Although neither age nor sex was significantly associated with B12 levels in univariate analysis, we also included these in the multivariable model. In those with MCI, AD and PD who had more than one B12 measurement, differences between the two measurements were calculated and divided by time elapsed to estimate median rates of decline for each group. These rates were compared using a non-parametric Kruskal-Wallis test. A significance threshold of 0.05 was used.

\section{Results \\ Vitamin B12 levels at baseline}

Of those diagnosed with predominant neurocognitive symptoms (AD, MCI, FTD, DLB), 16\% (220/1312) were excluded due to a $\mathrm{B} 12$ level $>911 \mathrm{pg} / \mathrm{ml}$. Of those evaluated for predominant parkinsonism (PD, MSA, PSP), $12 \%(84 / 691)$ were excluded due to having a B12 level > $911 \mathrm{pg} / \mathrm{ml}$. A total of 713 patients met criteria to be included in the analysis. Group characteristics across diagnostic categories were compared using ANOVA. Age and sex differed significantly across diagnostic groups (Table 1). B12 levels at time of diagnosis also varied significantly. Although patients with PD were more likely to have B12 levels $<250 \mathrm{pg} / \mathrm{ml}$, this difference was not significant. Neither use of a multivitamin nor a separate B12 supplement was different across groups. As expected, levodopa use was more common among those diagnostic groups with parkinsonism (PD, DLB, MSA, and PSP). The overall percentage of patients on metformin, a medication associated with a decline in B12, was low but use was significantly different across groups, with the DLB group having the greatest metformin use. Use of proton pump inhibitor was not significantly different across groups. (Table 1).

In univariate analyses examining the potential association of covariates with B12 levels around the time of disease diagnosis, levels of B12 in MCI, FTD, MCI and PSP were all found to be significantly higher on average than levels in those with PD (Table 2). No differences were observed between B12 levels in patients with PD and those with either AD or MSA. Neither age nor sex were associated with B12 levels, although the $95 \%$ CI for age indicated a higher likelihood of decreasing levels with increasing age. Both 
Table 1 Characteristics of cohort

\begin{tabular}{|c|c|c|c|c|c|c|c|c|}
\hline & $\mathrm{AD}(\boldsymbol{n}=204)$ & $\operatorname{DLB}(\boldsymbol{n}=36)$ & FTD $(\boldsymbol{n}=24)$ & $\mathrm{MCl}(\boldsymbol{n}=290)$ & $\mathrm{MSA}(\boldsymbol{n}=9)$ & $\mathrm{PD}(\boldsymbol{n}=130)$ & $\operatorname{PSP}(n=20)$ & $P$-value* \\
\hline $\begin{array}{l}\text { Age at diagnosis, mean } \\
\text { (sd) }\end{array}$ & $74.4(9.7)$ & $73.4(8.6)$ & $68.4(8.6)$ & $70.4(9.9)$ & 66.1 (3.6) & $68(10.2)$ & $69.9(6.3)$ & $<0.001^{* *}$ \\
\hline $\begin{array}{l}\text { Age at time of B12, } \\
\text { mean (sd) }\end{array}$ & $73.8(9.7)$ & $73(8.6)$ & $67.7(8.5)$ & $69.8(9.9)$ & $65.1(3.8)$ & $68.4(9.7)$ & $69(6.1)$ & $<0.001^{* *}$ \\
\hline Female (\%) & $118(57.8)$ & $17(47.2)$ & $11(45.8)$ & $150(51.7)$ & $3(33.3)$ & $51(39.2)$ & $9(45)$ & 0.04 \\
\hline $\begin{array}{l}\text { B12 level (pg/ml), } \\
\text { mean (sd) }\end{array}$ & 465.1 (165.5) & $518.7(180.4)$ & $542.9(159.3)$ & 499.4 (180.7) & $549.8(145.6)$ & $454.7(168.4)$ & $539.1(178.1)$ & $0.015^{* *}$ \\
\hline $\begin{array}{l}\text { Patients with B12 } \\
<250 \mathrm{pg} / \mathrm{ml}(\%)\end{array}$ & $13(6.4)$ & $2(5.6)$ & 0 & $18(6.2)$ & 0 & $13(10.0)$ & $1(5.0)$ & 0.69 \\
\hline \multicolumn{9}{|l|}{ Medications (\%) } \\
\hline Levodopa & $1(0.5)$ & $20(55.6)$ & 0 & 0 & $5(55.6)$ & $116(89.2)$ & $9(45)$ & $<0.001$ \\
\hline Multivitamin & $58(28.4)$ & $6(16.7)$ & $6(25)$ & $85(29.3)$ & $1(11.1)$ & $23(17.7)$ & $3(15.0)$ & 0.10 \\
\hline Vitamin B12 & $16(7.8)$ & $3(8.3)$ & $2(8.3)$ & $18(6.2)$ & 0 & $2(1.5)$ & $2(10.0)$ & 0.13 \\
\hline Metformin & $11(5.4)$ & $7(19.4)$ & $1(4.2)$ & $27(9.3)$ & 0 & $4(3.1)$ & $2(10.0)$ & 0.02 \\
\hline PP\| & $28(13.7)$ & $8(22.2)$ & $4(16.7)$ & 54 (18.6) & 0 & $16(12.3)$ & $5(25.0)$ & 0.27 \\
\hline
\end{tabular}

Abbreviations: $M C I$ mild cognitive impairment; $P D$ Parkinson's disease; $M S A$ multiple systems atrophy; DLB dementia with Lewy bodies; $A D$ Alzheimer's disease; $P S P$ progressive supranuclear palsy; FTD frontotemporal dementia; PPI proton pump inhibitor

"calculated using Fisher's exact test unless otherwise indicated

***alculated using analysis of variance

multivitamin use and B12 supplementation were significantly associated with increased B12 levels. Neither use of metformin or a proton pump inhibitor was associated with significantly lower B12 levels.

In multivariable analysis adjusted for sex, age, multivitamin use and B12 supplementation, patients with DLB, FTD and PSP all had higher B12 levels on average than those with $\mathrm{PD}$, although $\mathrm{MCI}$ was no longer significant $(p=0.068$; Table 3$)$. Multivitamin use and B12 supplementation were independently associated with increased B12 levels.

Table 2 Univariate linear regression of factors associated with B12 levels at time of diagnosis

\begin{tabular}{llll}
\hline Diagnosis & Coefficient & $(95 \% \mathrm{Cl})$ & P-value \\
\hline Parkinson's disease & Ref & & \\
Alzheimer's disease & 10.4 & $(-27.7,48.5)$ & 0.59 \\
Dementia with Lewy bodies & 63.9 & $(-0.06,128)$ & 0.05 \\
Frontotemporal dementia & 88.2 & $(12.7,164)$ & 0.02 \\
Mild cognitive impairment & 44.6 & $(9.77,80.5)$ & 0.015 \\
Multiple systems atrophy & 95.0 & $(-22.1,212)$ & 0.11 \\
Progressive supranuclear palsy & 84.4 & $(2.74,166)$ & 0.04 \\
Female gender & -9.56 & $(-35.2,16.1)$ & 0.47 \\
Age at diagnosis & -0.99 & $(-2.28,0.29)$ & 0.13 \\
Multivitamin use & 91.1 & $(62.4,120)$ & $<0001$ \\
B12 supplementation & 88.7 & $(35.3,142)$ & 0.001 \\
Metformin & 23.0 & $(-26.3,72.3)$ & 0.36 \\
Proton Pump Inhibitor & 0.62 & $(-34.2,35.5)$ & 0.97 \\
\hline
\end{tabular}

\section{Rate of vitamin B12 decline}

Of those diagnosed with predominant neurocognitive symptoms (AD, MCI, FTD, DLB), 16\% were excluded due to the first $\mathrm{B} 12>911 \mathrm{pg} / \mathrm{ml}$ and $23 \%$ due $>10 \%$ increase in the second B12 measurement compared to baseline. Of those evaluated for predominant parkinsonism, $14 \%$ were excluded due to the first B12>911 pg/ml and $27 \%$ due to an increase of $>10 \%$ at the second measurement. Since there are fewer patients with two B12 measurements, we confined the analysis of the

Table 3 Multivariable analysis of factors associated with B12 level at time of diagnosis

\begin{tabular}{llll}
\hline Diagnosis & Coefficient $^{*}$ & $(95 \% \mathrm{Cl})$ & P-value \\
\hline Parkinson's disease (PD) & Ref & & \\
Alzheimer's disease (AD) & 1.90 & $(-36.4,40.2)$ & 0.92 \\
Dementia with Lewy bodies (DLB) & 64.3 & $(1.94,127)$ & 0.04 \\
Frontotemporal dementia (FTD) & 76.4 & $(3.24,149)$ & 0.04 \\
Mild cognitive impairment (MCI) & 32.8 & $(-2.39,68.0)$ & 0.068 \\
Multiple systems atrophy (MSA) & 100.4 & $(-12.8,214)$ & 82 \\
Progressive supranuclear palsy (PSP) & 81.6 & $(2.55,161)$ & 0.04 \\
Female gender & -7.65 & $(-32.6,17.3)$ & 0.55 \\
Age at diagnosis & -0.86 & $(-2.14,0.41)$ & 0.185 \\
Multivitamin use & 93.5 & $(65.0,122)$ & $<0.001$ \\
B12 supplementation & 86.4 & $(34.3,139)$ & 0.001 \\
\hline
\end{tabular}

* In a linear regression, coefficients are interpreted as follows: a one unit increase in $\mathrm{x}$ results in an increase in $\mathrm{B} 12 \mathrm{pg} / \mathrm{ml}$ of (coefficient) pgs. For example, the coefficient associated with DLB is interpreted: Compared to patients with PD, patients with DLB have on average a B12 level that is 64.3 $\mathrm{pg} / \mathrm{ml}$ higher at the time of diagnosis 
change in $\mathrm{B} 12$ level/year across the $\mathrm{PD}, \mathrm{MCI}$ and $\mathrm{AD}$ groups, and a total of 69 patients were included in the analysis (Fig. 1). Median (interquartile range) rate of decline for those with $\mathrm{PD}, \mathrm{MCI}$, and $\mathrm{AD}$ were $-17(-35$, $-0.4),-47(-112,-8)$, and $-29(-124,2.4) \mathrm{pg} / \mathrm{ml} /$ year, respectively. Rate of decline did not differ significantly across groups.

\section{Discussion}

In this cohort of patients with neurodegenerative conditions, we found that B12 levels obtained within 3 years of neurological diagnosis were significantly lower in patients with PD than in patients with PSP, FTD, and DLB when adjusted for sex, age, and B12-supplementing medications. The rate of $\mathrm{B} 12$ decline in $\mathrm{PD}, \mathrm{MCI}$, and $\mathrm{AD}$ ranged from 17 to $47 \mathrm{pg} / \mathrm{ml} /$ year, far in excess of the approximately 5 $\mathrm{pg} / \mathrm{ml} /$ year measured in a study of normal elderly adults [9]. These findings are of clinical importance since we have previously found an association of low B12 levels with more rapid development of gait impairment in PD [7] but is also relevant in patients with other neurodegenerative diseases, who are expected to develop cognitive impairment and/or gait instability due to disease progression. Failure to identify and treat this deficiency at diagnosis or in the years after diagnosis may well increase patient morbidity.

As expected, B12 supplementation and multivitamin use were associated with higher B12 levels. Multivitamin use ranged from 11 to $29 \%$ while separate B12 supplement use ranged from $1.5-10 \%$. These rates were similar to the rates of use of about 41 and 5\%, respectively, in a recent study of a large PD cohort [10] and were not statistically different across groups. Both the age of diagnosis and percentage of females were lower in PD and MSA, but linear regression models showed that age and sex were not associated with B12 levels.

One of the limitations of this study is that we did not have information on patient body mass index (BMI) and nutrition risk index. Obesity and elevated BMI have been shown to be associated with lower vitamin B12 levels [11], whereas in hospitalized patients at nutritional risk, high B12 levels (>1000 pg/nl) have been shown to be associated with increased mortality [12]. In contrast, our study targeted outpatients with early neurodegenerative disease excluded patients with levels over $911 \mathrm{pg} / \mathrm{ml}$, and is therefore was different patient population. However, since patients with advanced neurodegenerative diseases are at higher risk for impaired nutritional status, future studies of moderately advanced neurodegenerative disease should evaluate associations of B12 with mortality.

A systematic literature review testing for an association of low vitamin B12 levels with development of cognitive impairment has been inconclusive [13], although studies using more specific biomarkers of low B12 status (methylmalonic acid and holotranscobalamin) have shown significant associations [14]. While in our study, the B12 levels were lower and comparable in PD and AD patients, Boston et al. (2019) have shown that vitamin B12 levels were not significantly lower in $\mathrm{AD}$ patients compared to healthy controls [15]. One reason for the apparent difference in our findings may be that we only looked at patients within 3 years of AD diagnosis. Our study also lacks a healthy control group for comparison.

In PD particularly, lower B12 levels might be more likely to develop for a number of reasons, including change in dietary intake of B12 (some adopt a lowprotein diet), Helicobacter pylori infection, delayed gastric emptying and development of constipation, which is

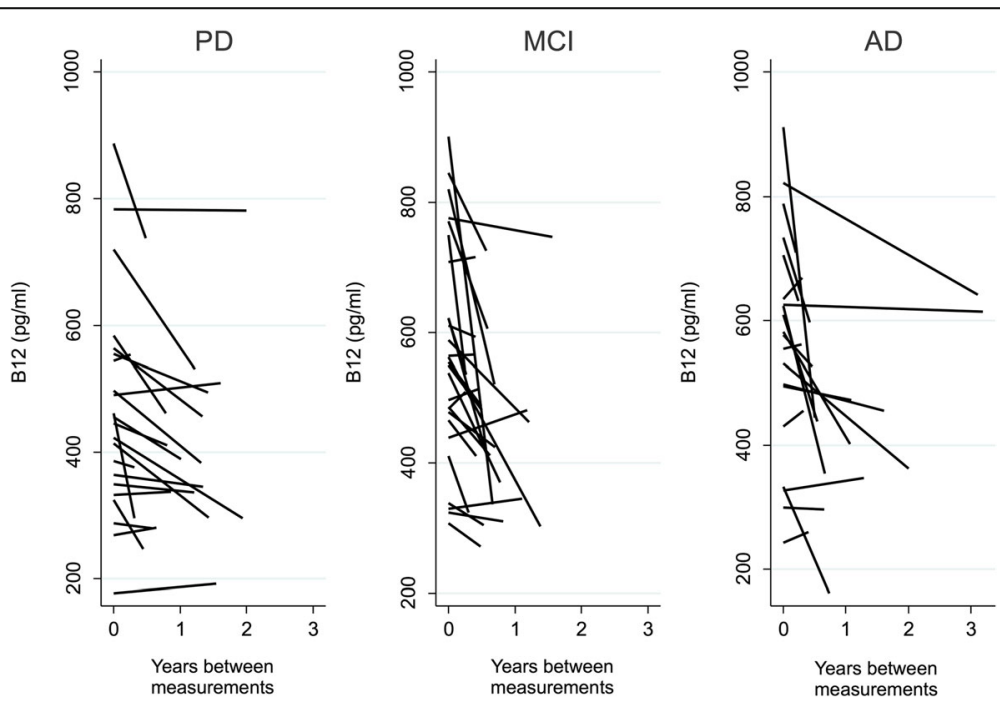

Fig. 1 Rate of $B 12$ Decline in PD, MCl, and AD patients. Plots showing individual declines of B12 in patients with PD, MCl, and AD. Median (interquartile range) rate of decline were $-17(-35,-0.4),-47(-112,-8)$, and $-29(-124,2.4) \mathrm{pg} / \mathrm{ml} /$ year for PD, $M C l$, and $A D$, respectively 
associated with bacterial overgrowth [16]. Finally, the prevalence of borderline low B12 $(<250 \mathrm{pg} / \mathrm{ml})$ in $10 \%$ of PD patients in this cohort from 2007 to 2015 was similar to what was observed in the DATATOP study (13\%) [6], suggesting that it remains a fairly common comorbidity in PD.

Our prior study showing that low B12 levels predicted greater progression of gait instability did not demonstrate similar predictive characteristics associated with other B12 analytes traditionally used to establish a diagnosis of B12 deficiency, including methylmalonic acid, homocysteine and holotranscobalamin [7]. This observation raises the question as to whether B12 levels might have a more direct effect on PD progression. Interestingly, Schaffner and colleagues recently reported that B12 directly modulates leucine-rich repeat kinase 2 (LRRK2) [17]. Mutations in LRRK2 account for about 5\% of hereditary PD [18] and the most common variant (G2019S) has been established to increase LRRK2 activity $[19,20]$. Since Schaffner and colleagues found that higher levels of B12 inhibit LRRK2 kinase activity, their work suggests that low B12 levels may directly contribute to PD pathogenesis by allowing for increased LRRK2 activity [21].

While aggregation of specific proteins ( $\alpha$-synuclein and $\beta$-amyloid) are defining characteristics PD and AD pathology, the role of these proteins in the early pathogenesis of the sporadic forms of these disorders is not clear and has led some to hypothesize that insight into disease modifying targets requires discovery of other biomarkers present at disease onset and subsequent subgroup analysis according to these candidate biomarkers [22, 23]. Data from this study, showing that B12 levels are lower in PD than other neurodegenerative diseases, along with recent clinical [7] and biochemical data [17] suggest that B12 warrants further study as an early PD biomarker.

Of note in hospitalized patients, high vitamin B12 levels have been associated with increased in-hospital mortality [12, 24-26]. This may be because cobalamin is an acute phase reactant, and elevated cobalamin levels have been reported in patients with oncologic diseases, hepatic disease, and in the elderly, hospitalized and critically ill medical patients who were not taking B12 supplements $[12,27]$. Since it is unknown whether there is a relationship between high vitamin B12 levels and mortality in patients with neurodegenerative disorders, future studies should explore this question.

To our knowledge, this is the largest study to assess B12 levels across early neurodegenerative diseases. However, our study is limited by its observational design and small sample size for the less common disorders. Because the study was retrospective and was based in the Movement Disorder and the Behavioral Neurology clinics at UCSF, a suitable control group with B12 measurements was not available. Although B12 measurements are commonly obtained in the initial evaluation of patients with gait or cognitive conditions, in this study B12 measurements were drawn as part of usual clinical practice. It is therefore possible that initial or repeat B12 levels were more likely to be measured in those who developed neuropathy, possibly causing ascertainment bias and/or an overestimate of annualized decline in B12 levels. Also, since supplement use was based on retrospective chart review, this information is likely to be less accurate than that collected as part of a prospective study. Finally, although we were not able to correlate disease stage with B12 levels, because the analysis was restricted to patients with B12 levels measured within 3 years of diagnosis, these patients were predominantly in early disease stages.

\section{Conclusion}

Our finding of a lower B12 level around the time of diagnosis in PD compared to PSP, FTD, and DLB and greater rates of $\mathrm{B} 12$ decline in those with $\mathrm{PD}, \mathrm{AD}$ and $\mathrm{MCI}$ supports the need for increased vigilance for B12 deficiency in these conditions. Future studies are needed to confirm these findings and determine whether low B12 is associated with more rapid disease progression and if so, whether early B12 supplementation reduces morbidity.

\section{Abbreviations}

AD: Alzheimer's disease; DLB: Dementia with Lewy Bodies;

FTD: Frontotemporal Dementia; LRRK2: Leucine-rich repeat kinase 2; MCl: Mild Cognitive Impairment; MSA: Multiple System Atrophy;

PD: Parkinson's disease; PSP: Progressive Supranuclear Palsy; UCSF: University of California, San Francisco

\section{Acknowledgements}

Not applicable.

\section{Authors' contributions}

$\mathrm{NL}$ and CC made substantial contributions to conception, design, analysis and interpretation of data, drafting the manuscript and gave final approval of the version to be published. AM made substantial contributions to acquisition of data and review of manuscript. NH made substantial contributions to statistical analysis, interpretation of data, and review of manuscript. Each author has participated sufficiently in the work to take public responsibility for appropriate portions of the content and agreed to be accountable for all aspects of the work in ensuring that questions related to the accuracy or integrity of any part of the work are appropriately investigated and resolved. All authors read and approved the final manuscript.

\section{Funding}

This study was funded by unrestricted grants from the Margaretten, Tsu, and Liu families and by the National Center for Advancing Translational Sciences, National Institutes of Health, through UCSF-CTSI Grant Number UL1

TR000004. Its contents are solely the responsibility of the authors and do not necessarily represent the official views of the $\mathrm{NIH}$.

Availability of data and materials

The data that support the findings of this study are available from the authors upon reasonable request.

Ethics approval and consent to participate

This retrospective study was reviewed and approved by the Committee on Human Research at UCSF and was carried out in accordance with their ethical standards and regulations. 


\section{Consent for publication}

Not applicable, as the manuscript does not contain any individual person's data.

\section{Competing interests}

The authors declare that they have no competing interests.

\section{Author details}

'Department of Neurology, University of California, San Francisco, 1635 Divisadero, Suite 520-530, San Francisco, CA 94115, USA. ${ }^{2}$ Department of Neurology, University of California, San Francisco, 400 Parnassus Avenue, San Francisco, CA 94122, USA. ${ }^{3}$ Department of Epidemiology and Biostatistics, University of California, San Francisco, 550 16th Street, San Francisco, CA 94158, USA.

\section{Received: 8 November 2019 Accepted: 4 March 2020}

Published online: 12 March 2020

\section{References}

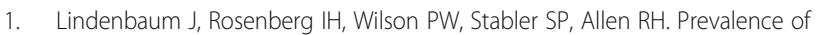
cobalamin deficiency in the Framingham elderly population. Am J Clin Nutr. 2015;60:2-11.

2. Vogiatzoglou A, Smith AD, Nurk E, Drevon CA, Ueland PM, Vollset SE, et al. Cognitive function in an elderly population: interaction between vitamin B12 status, depression, and apolipoprotein E ع4: the Hordaland Homocysteine study. Psychosom Med. 2013;75:20-9.

3. Stabler S. Vitamin B12 deficiency. NEJM. 2013;68:149-60.

4. Hin H, Clarke R, Sherliker P, Atoyebi W, Emmens K, Birks J, et al. Clinical relevance of low serum vitamin B12 concentrations in older people: the Banbury B12 study. Age Ageing. 2006:35:416-22

5. Kim JM, Stewart R, Kim SW, Shin IS, Yang SJ, Shin HY, et al. Changes in folate, vitamin B12 and homocysteine associated with incident dementia. J Neurol Neurosurg Psychiatry. 2008;7:864-8.

6. Parkinson's Study Group. Effects of tocopherol and deprenyl on the progression of disability in early Parkinson's disease. NEJM. 1993;328:176-83.

7. Christine CW, Auinger P, Joslin A, Yelpaala Y, Green R, Parkinson study group-DATATOP investigators. Vitamin B12 and homocysteine levels predict different outcomes in early Parkinson's disease. Mov Disord. 2018;33:762-70.

8. Parashos SA, Elm J, Boyd JT, Chou KL, Dai L, Mari Z, et al. Validation of an ambulatory capacity measure in Parkinson disease: a construct derived from the unified Parkinson's disease rating scale. J Park Dis. 2015;5:67-73.

9. Nilsson-Ehle H, Jagenburg R, Landahl S, Lindstedt S, Svanborg A, Westin J. Serum cobalamins in the elderly: a longitudinal study of a representative population sample from age 70 to 81. Eur J Haematol. 1997;47:10-6.

10. Dietiker C, Kim S, Zhang Y, Christine CW. Characterization of vitamin B12 supplementation and correlation with clinical outcomes in a large longitudinal study of early Parkinson's disease. J Mov Disord. 2019;12:91-6.

11. Sun Y, Sun M, Liu B, Du Y, Rong S, Xu G, Snetselaar LG, Bao W. Inverse Association Between Serum Vitamin B12 Concentration and Obesity Among Adults in the United States. Front Endocrinol (Lausanne). 2019;10:414.

12. Cappello S, Cereda E, Rondanelli M, Klersy C, Cameletti B, Albertini R, Magno D, Caraccia M, Turri A, Caccialanza R. Elevated plasma vitamin B12 concentrations are independent predictors of in-hospital mortality in adult patients at nutritional risk. Nutrients. 2016;9(1):1.

13. O'Leary F, Allman-Farinelli M, Samman S. Vitamin $B_{12}$ status, cognitive decline and dementia: a systematic review of prospective cohort studies. $\mathrm{Br}$ J Nutr. 2012;108:1948-61.

14. Lildballe DL, Fedosov S, Sherliker P, Hin H, Clarke R, Nexo E. Association of cognitive impairment with combinations of vitamin $B_{12}$-related parameters. Clin Chem. 2011;57:1436-43.

15. Boston PF, McKirdy SJ, Al-Turki MA, Barker ME, Russell JM. Vitamin B12 and folate levels in progression of Alzheimer's disease - a short report. Int J Psychiatry Clin Pract. 2019;29:1-3.

16. Fasano A, Visanji N, Liu L, Lang A, Pfeiffer R. Gastrointestinal dysfunction in Parkinson's disease. Lancet Neurol. 2015;14:625-39.

17. Schaffner A, Li X, Gomez-Llorente Y, Leandrou E, Memou A, Clemente N. Vitamin $B_{12}$ modulates Parkinson's disease LRRK2 kinase activity through allosteric regulation and confers neuroprotection. Cell Res. 2019;29:313-29.

18. Healy DG, Falchi M, O'Sullivan SS, Bonifati V, Durr A, Bressman S. Phenotype, genotype, and worldwide genetic penetrance of LRRK2-associated Parkinson's disease: a case-control study. Lancet Neurol. 2008;7:583-90.
19. West AB, Moore DJ, Biskup S, Bugayenko A, Smith WW, Ross CA, et al. Parkinson's disease-associated mutations in leucine-rich repeat kinase 2 augment kinase activity. Proc Natl Acad Sci. 2005;102:16842-7.

20. Sheng Z, Zhang S, Bustos D, Kleinheinz T, Le Pichon CE, Dominguez SL, et al. Ser1292 autophosphorylation is an indicator of LRRK2 kinase activity and contributes to the cellular effects of PD mutations. Sci Transl Med. 2012;164:164ra161.

21. Green R, Christine CW. Linking vitamin B12 and a trembling disorder. Cell Res. 2019;29:343-4.

22. Espay AJ, Schwarzschild MA, Tanner CM, Fernandez HH, Simon DK, Leverenz $\mathrm{JB}$, et al. Biomarker-driven phenotyping in Parkinson's disease: a translational missing link in disease-modifying clinical trials. Mov Disord. 2017;32(3):319-24.

23. Espay AJ, Vizcarra JA, Marsili L, Lang AE, Simon DK, Merola A, et al. Revisiting protein aggregation as pathogenic in sporadic Parkinson and Alzheimer diseases. Neurology. 2019;92:329-37.

24. Hemmersbach-Miller M, Conde-Martel A, Betancor-Leon P. Vitamin B12 as a predictor of mortality in elderly patients. J Am Geriatr Soc. 2005;53:2035-6.

25. Salles N, Herrmann F, Sakbani K, Rapin CH, Sieber C. High vitamin B12 level: A strong predictor of mortality in elderly inpatients. J Am Geriatr Soc. 2005; 53:917-8.

26. Baztan JJ, Gavidia JJ, Gomez-Pavon J, Esteve A, Ruiperez I. High vitamin B12 levels and in-hospital mortality. J Am Geriatr Soc. 2010;58:2237-8.

27. Arendt JF, Nexo E. Cobalamin related parameters and disease patterns in patients with increased serum cobalamin levels. PLoS One. 2012;7:e45979.

\section{Publisher's Note}

Springer Nature remains neutral with regard to jurisdictional claims in published maps and institutional affiliations.

\section{Ready to submit your research? Choose BMC and benefit from:}

- fast, convenient online submission

- thorough peer review by experienced researchers in your field

- rapid publication on acceptance

- support for research data, including large and complex data types

- gold Open Access which fosters wider collaboration and increased citations

- maximum visibility for your research: over $100 \mathrm{M}$ website views per year

At BMC, research is always in progress.

Learn more biomedcentral.com/submissions 